\title{
A Geovisual Analytics Approach for Mouse Movement Analysis
}

\author{
Ali Tahir \\ School of Computer Science and Informatics \\ University College Dublin \\ Belfield, Dublin 4, Ireland \\ E-mail: ali.tahir@ucd.ie
}

\section{Gavin McArdle}

National Centre for Geocomputation

National University of Ireland Maynooth

Maynooth, Co. Kildare, Ireland

E-mail: gavin.mcardle@nuim.ie

\section{Michela Bertolotto}

School of Computer Science and Informatics

University College Dublin

Belfield, Dublin 4, Ireland

E-mail: michela.bertolotto@ucd.ie

\begin{abstract}
The use of Web maps has created opportunities and challenges for map generation and delivery. While volunteered geographic information has led to the development of accurate and inexpensive Web maps, the sheer volume of data generated has created spatial information overload. This results in difficulties identifying relevant map features. Geopersonalisation, which adapts map content based on user interests offers a solution to this. The technique is especially powerful when implicit indicators of interest are used as a basis for personalisation. This article describes the design and features of VizAnalysisTools, a suite of tools to visualise and interpret users' implicit interactions with map content. While traditional data mining techniques can be used to identify trends and preferences, visual analytics, in particular Geovisual Analytics, which assists the human cognition process, has proven useful in detecting interesting patterns. By identifying salient trends, areas of interest on the map become apparent. This knowledge can be used to strengthen the algorithms used for Geopersonalisation.
\end{abstract}

Copyright (c) 200x Inderscience Enterprises Ltd. 
Keywords: geographical information systems; Geovisual Analytics; geovisual analysis; Geoweb; web GIS; Geopersonalisation; Geovisualisation; GIS; web architecture; map personalisation; web mapping; heat map; speed map; mouse movements; open source.

Reference to this paper should be made as follows: Tahir, A., McArdle, G. and Bertolotto, M. (xxxx) 'A Geovisual Analytics Approach for Mouse Movement Analysis', Int. J. of Data Mining, Modelling and Management, Vol. x, No. x, pp.xxx-xxx.

Biographical notes: Ali Tahir is a postgraduate research student in the School of Computer Science in Informatics at University College Dublin since 2009. He received his BSc degree in Computing and Information Systems in 2004 from Oxford Brookes University, UK and subsequently a MSc degree in Geographical Information Science from University of Nottingham, UK in 2006. Afterwards, he has worked as Junior Lecturer in National University of Sciences and Technology (NUST) in Pakistan until 2009. His research activities include Geovisualisation, Web GIS, Geopersonalisation.

Dr. Gavin McArdle received a BSc degree in Computer Science in 2003 from University College Dublin (UCD). He subsequently completed his $\mathrm{PhD}$ degree there in 2008, where he investigated the benefits of using virtual reality environments for e-learning. In June 2008 Gavin began a postdoctoral research position in UCD, where he investigated map personalisation by utilising user preferences. Since June 2010, Gavin has been a postdoctoral research fellow at the National Centre for Geocomputation at NUI, Maynooth, examining visual techniques for analysing large movement datasets. Gavin's research interests include geo-visualisation, personalisation techniques and location-based services.

Dr. Michela Bertolotto received a $\mathrm{PhD}$ in Computer Science from the University of Genoa (Italy) in 1998. Subsequently she worked as a Postdoctoral Research Associate in the Nation Center for Geographic Information and Analysis (NCGIA) at the University of Maine. Since 2000 she has been a faculty member at the School of Computer Science and Informatics of University College Dublin. Her research interests include web-based and wireless GIS, spatio-temporal data modelling, and $3 \mathrm{D}$ data handling and interfaces.

\section{Introduction}

The last decade has witnessed huge progress in the accessibility of spatially referenced information primarily because of the increasing mobility of individuals and advances in ubiquitous technologies such as Global Positioning Systems (GPS). Furthermore, Web mapping systems such as Google Maps (maps.google. 
com), Google Earth (google.com/earth) and Bing Maps (bing.com/maps), as well as repositories such as OpenStreetMap (openstreetmap.org) permit end users to contribute to their datasets.

This growth in data availability has led to spatial information overload where the sheer volume of data provided on a map makes it difficult for a user to extract meaningful information. This has driven the demand for more sophisticated and personalised Web map applications.

Geopersonalisation is a recent development which contributes to resolving geospatial information overload by adapting a map to reflect user preferences and interests. To achieve this, both explicit and implicit approaches can be utilised to determine interests. The former directly solicits preferences from the user, often through a series of questions, while the latter gathers the information automatically during a user session by monitoring interactions with the application. While direct questions produce accurate results, implicit monitoring places less burden on the user and has a 100 percent completion rate. A recent study in Geopersonalisation shows that implicitly monitoring mouse movements produces a reliable indicator of interest with spatial data (Mac Aoidh et al., 2009). In this approach, a user profile is generated based on this knowledge. Personalised maps are then produced matching these profiles by promoting appropriate content while removing less appealing content (Ballatore et al., 2010).

User interactions can also be visually analysed to gain a greater insight into user behaviours by exploring spatial thinking and spatial cognition phenomena. The research presented in this article employs Geovisual Analytics (Andrienko, Andrienko, Jankowski, Keim, Kraak, MacEachren and Worbel, 2007) to reveal patterns and trends in user interactions with digital maps. Particular attention is given to mouse movement analysis. Using Geovisualisation techniques such as heat map and speed map, applied to mouse movements in the form of map clicks, mouse hesitations and mouse trajectories, hot spots and user familiarity with the area can be identified. In addition to the support of Geovisual Analytics, the tool we have developed VizAnalysisTools employs Web 2.0 technology (Oreilly, 2007), a powerful extension of the Web that presents interactive information sharing and promotes interoperability. VizAnalysisTools can be used to analyse mouse movement datasets to reveal how users perform specific spatial choices as well as understanding Human-Computer Interactions (HCI) when using Geographical Information Systems (GIS). This can be used to achieve a better form of Geopersonalisation and user profiling techniques (Tahir et al., 2010).

Our previous work described a Web architecture for Geopersonalisation that utilises open source technologies (McArdle et al., 2010), conforming to Open Geospatial Consortium (OGC) standards. The architecture has now been augmented with a Geovisual Analytics component to analyse mouse movements through their visualisation on the underlying map data. Spatio-temporal analysis and summary statistics are also included. Visualisation of user interactions is achieved as Keyhole Markup Language (KML) overlays while for statistical reports and charts, Google Visualisation API is embedded within the Web interface. A clustering component has also been incorporated in our architecture.

The research presented here builds upon preliminary work (Tahir et al., 2011), which was extended to include a complete case study of our application. The 
remainder of this paper is organised as follows. Section 2 outlines the related work in the field. Section 3 describes the system and its functionality by providing some examples of the tool in use. The system architecture and the associated technologies are discussed in Section 4. A case study, which demonstrates how the system can be used to identify intentions and interests, is presented in Section 5 . Finally concluding comments and directions for future work are presented in Section 6 .

\section{Related Work}

An extensive amount of research has been carried out in the area of Geovisualisation. However in the context of Geopersonalisation, this field has drawn less attention. Previous studies in Geopersonalisation describe user interactions in the form of mouse movements on spatial interfaces. For example, Mac Aoidh et al. (2008) performed preliminary work for visually analysing mouse movement data and developed a research prototype called Geospatial Interactions Visualizer (GIViz). This stand-alone application analysed mouse positions and clicks from a single user rather than mouse trajectories recorded on multiple map scales.

Other tools have been developed to support Geovisualisation tasks in multidisciplinary domains. CommonGIS is a case in point (Andrienko, Andrienko and Wrobel, 2007). CommonGIS can visually analyse generic movement datasets through a range of spatio-temporal analysis tools (including space-time cube, time aware maps, time series charts). While our tool, VizAnalysisTools, and CommonGIS both provide analysis of spatial and spatio-temporal data, there are key differences in how this is achieved and the level of granularity offered. For example, CommonGIS is limited to a stand-alone application where the user has to install software packages before using the tool, while VizAnalysisTools uses Web-service technology and is therefore platform independent. Furthermore, VizAnalysisTools specifically supports developers through the visualisation of spatial usage behaviour and interaction patterns of end users, with a view to using this information to improve the mapping systems. This is similar to the way Google

Analytics (google.com/analytics) assists developers to produce user centred websites.

Geovisualisation tools are derived from the more general information visualisation discipline. The advancement of multidisciplinary visualisation domains led to a new research area, Visual Analytics, which is defined as the science of analytical reasoning facilitated by visual interactive interfaces (Thomas and Cook, 2005). Visual Analytics has emerged as a result of the combined effect of information visualisation and scientific visualisation fields. Recently, Chen (2010) argued that, Geovisualisation, software visualization, and Visual Analytics have become specialised fields to deal with information visualisation in their respective domains. Within this arena, a new research discipline specifically targeted towards the spatio-temporal domain called Geovisual Analytics (Andrienko, Andrienko, Jankowski, Keim, Kraak, MacEachren and Worbel, 2007) has emerged.

Besides Geovisual Analytics, exploratory visualisation techniques have also become very prominent in the spatial domain in recent years. A heat map is such a 
technique that has become popular on the Web, where the colour intensity shows the amount of interaction that took place on a particular section of the Web page. For example MouseTrack (Arroyo et al., 2006) and Cheese (Mueller and Lockerd, 2001) are two systems that visualise non-spatial user interactions with the help of heat maps. Kisilevich et al. (2010) present interactive visualisations based on heat maps of exploration of attractive places for the tourists. The authors make use of Flickr (flickr.com) and Panoramio (panoramio.com) photos to identify interest of geographical areas.

The research presented in this paper describes a Geovisual Analytics Web tool which utilises open Web services conforming to OGC standards in order to build a shared and accessible platform. The approach improves upon existing systems for analysing mouse movements by incorporating a Web-based dimension and also by providing an increased range of spatio-temporal analysis options equipped with Geovisual Analytics. The robust and open nature of the tool allows it to be extended to incorporate other movement datasets in the future to resolve geospatial information overload challenges.

\section{VizAnalysisTools}

VizAnalysisTools is a suite of tools to analyse mouse movements and identifying usage patterns and behaviours which indicate important user intentions and interests.

The prototype has been deployed as a Web application which shows details of a user session. Each user session includes the interaction history of the user. The interface is delivered via a Web page which has two principal components. One component renders overlays of different interactions with a Web map, while a second component provides a statistical report of user interaction.

A typical user session on the Web interface highlights mouse hesitations and map clicks as an overlay, rendered using the KML format. These user interactions show the spatial interests of the user in question.

Bounding boxes, also called interaction windows, show user interests in certain areas of the map. When a user pans or zooms, a new bounding box is generated. For example, if a user zooms in on the map, we can conclude that they are more interested in that area of the map. As a result when examining interactions in that area, more weight should be given to the findings. The concept is shown in Figure 1, where several bounding boxes are rendered in black as a KML overlay on the base map. The bounding boxes shown on the map correspond to the number of zoom and pan operations the user made while studying the map. The corresponding reporting interface on the right hand side of the screen shot shows a statistical analysis of the bounding box usage. This interface consists of a bar chart showing map bounds on various scales. The $x$-axis shows the number of bounding boxes as the user zooms and pans, while the $y$-axis shows the map scale. This reporting provides analysts with an overview of the user interactions that took place on different scales. Any bar in the chart can be clicked to move to a particular scale on the map as the chart and map are linked interactively. Mouse speed can also be visualised using VizAnalysisTools. When considered alongside the trajectory of the mouse, the relative acceleration and deceleration of the cursor 


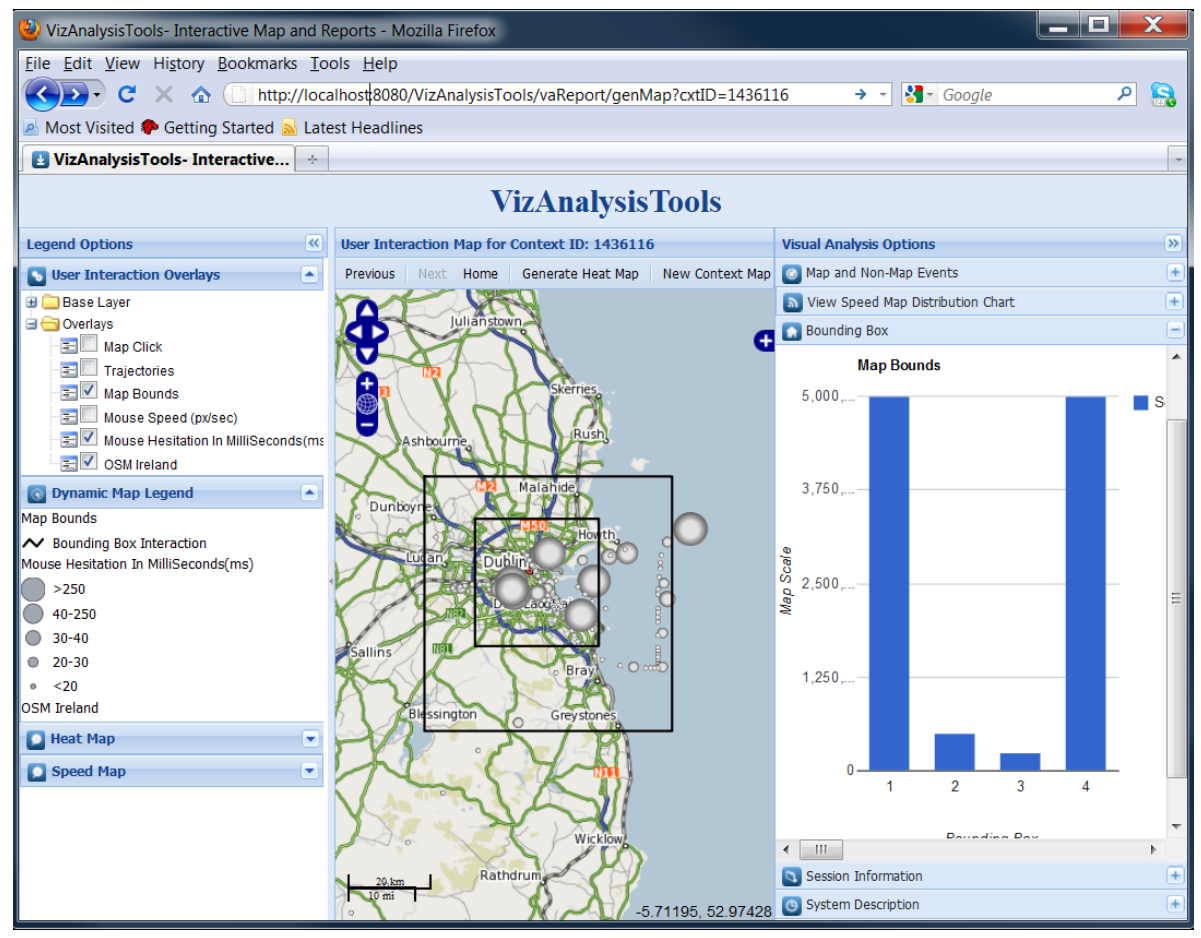

Figure 1 User interactions shown within bounding boxes and the corresponding bar chart

reveal a change in user behaviour which may indicate a shift in their intentions (Mac Aoidh et al., 2008). This functionality has been incorporated into the Web tool by categorising the mouse speed measured in pixels per second ( $\mathrm{px} / \mathrm{sec}$ ). Each speed category is assigned a different colour ranging from a minimum to a maximum speed value as shown in Figure 2.

The corresponding reporting interface in Figure 2 shows a speed chart which gives information about the generated speed map. The speed distribution shows a column chart which plots the speed range categories against the number of mouse movements. The trend shows that most of the time mouse movements were observed in the first speed range $(0.0$ to $2.0 \mathrm{px} / \mathrm{sec})$. The speed categories are dynamically generated based on the minimum, average and maximum speed of a particular user interaction. By visualising theses trends of the same user over multiple sessions, the average mouse speed can highlight the habits of the users and their level of interactions for example beginner or advanced users.

Similarly, a heat map is another visual technique which is generated based on user mouse interactions and rendered as an overlay on the base map. This visualisation allows analysts to identify areas of interest and where most interaction took place on the map. The amount of interaction corresponds to the amount of heat generated as a result. Importantly, an analyst can zoom in and out to see how the relative heat changes at different map scales for a more detailed insight into certain areas of the map. 


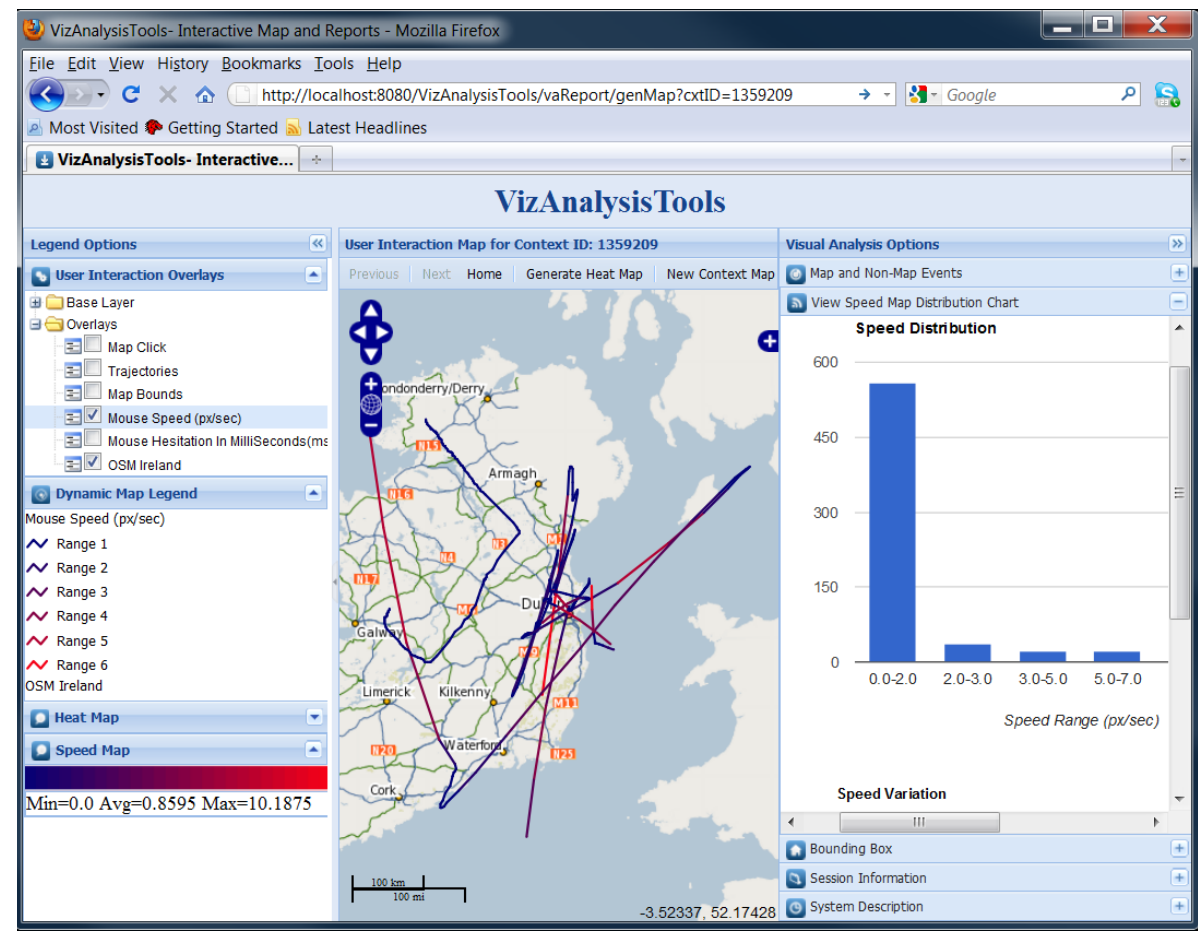

Figure 2 Mouse speed and corresponding speed distribution chart

When the features described above are used in conjunction with each other, this allows the analyst to reveal trends and patterns in the behaviours of users. Such patterns would be difficult to detect using data mining approaches alone. It is the domain knowledge of the analysts which adds a useful insight into interpreting the interactions which the tool produces.

\section{System Architecture and Technologies}

The system architecture of VizAnalysisTools is an extension of a Web architecture as described by McArdle et al. (2010), which employs open source interoperable technologies. VizAnalysisTools provides components which utilise the dynamics of interactive JavaScript API's and the core business logic to perform visual analysis and geo-computation using a visualisation and clustering engine on the server side. The architecture is based on Grails (grails.org), which is an open source framework to develop dynamic and robust Web applications. The system architecture is shown in Figure 3 and the core components of the architecture are described below.

Web services have emerged as a framework to exchange information over the Web between applications using Extensible Markup Language (XML). The Web service framework specifications include communication protocols such as Simple Object Access Protocol (SOAP), service descriptions such as the 


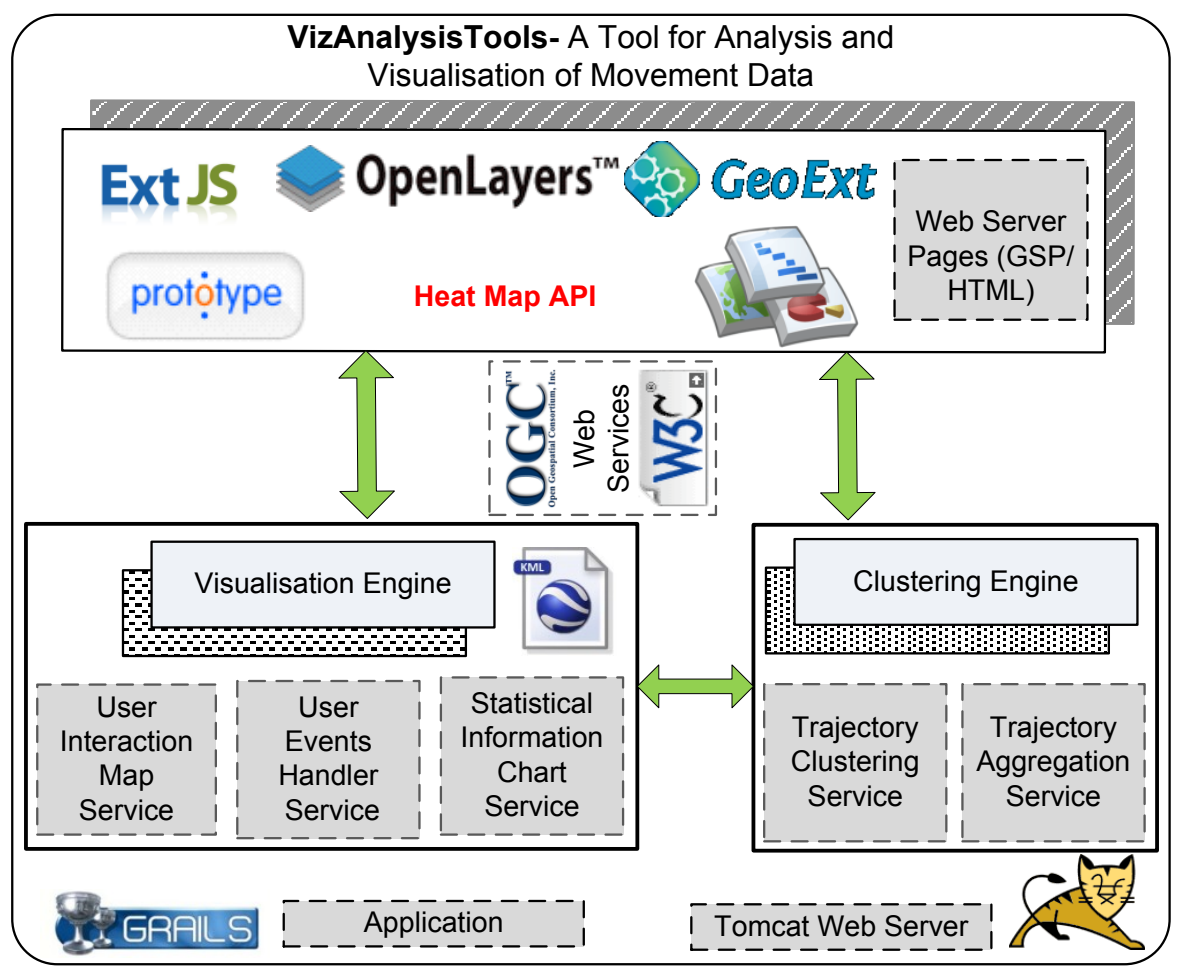

Figure 3 The system architecture

Web Services Description Language (WSDL), and service discovery such as Universal Description, Discovery and Integration (UDDI) (Curbera et al., 2002). VizAnalysisTools requests data from the system described in McArdle et al. (2010), which is an application to log and record user interactions.

The exchange of information takes place using SOAP, which returns an XML list of events for a particular user session. A user session that includes the interaction history is returned as an XML file. This information is passed to the visualisation engine which generates the map overlays and produces the statistical reports. Once the data is received in response to the Web service request by the visualisation engine, it starts processing this information using an internal KML service. This service parses the data and generates various KML files describing user interactions. KML (opengeospatial.org/standards/kml) is an OGC standard whose data is mainly composed of geographic visualisations including annotation of maps and images.

The structure of the generated KML file name, for example (localhost:8080/ VizAnalysisTools/file/mousespeed_1436116.kml) is a Universal Resource Identifier (URI), which in turn is an AJAX call to request a KML file of a particular type (merged with its context number, session number or user name). Each user interaction is assigned a context which holds all the information about the user interaction and the type of interaction (e.g., mapclick, mousespeed, 
mousehesitation, mousemove, mouseheat, mousetrajectory). This URI structure has been adopted using the Linked Data principles as described by Auer et al. (2009). This approach adds a new dimension to the data Web where this structure can be used in a shared and integrated environment with other applications. After the KML structure is formalised, it can be used to render overlays on the base map.

Various technologies are used to process the data in order to generate the map and statistical visualisations. For example, OpenLayers (openlayers.org) is an open source JavaScript library for displaying and manipulating spatial data in Web browsers, without server-side dependencies and is used to build rich Webbased geographic applications. OpenLayers widely supports OGC standards such as Web Map Service (WMS) and KML which are used in VizAnalysisTools to render the background OpenStreetMap layer (openstreetmap.org) and dynamic overlays respectively. The heat map described in section 3 is generated using Heat Map, a client side JavaScript library which works in conjunction with OpenLayers. This API inputs geographical locations and outputs intensity in different colours. The actual heat map is then generated as an overlay on the base map to show areas of interest.

GeoExt (geoext.org), which also operates with OpenLayers, provides a powerful way of developing highly interactive and dynamic Web GIS applications with the use of JavaScript. With GeoExt, ExtJS is used to build and design the layout which consists of a map, dynamic legends, and interactive visual analysis options. The advantage of using ExtJS dynamic layouts is that more functionality can be embedded irrespective of the available screen space. For this project, GeoExt has been used extensively for rendering the spatial and non-spatial features of the interactive interface.

Prototype (prototypejs.org) is a framework that is used with the JavaScript development in the Web environment. VizAnalysisTools uses Prototype to implement the system functionality and to generate the interaction maps and reporting interface. The dynamic reporting interface, provided within the Web interface, produces charts summarising the main interactions. This is achieved using the Google Visualisation API (code.google.com/apis/charttools). The API is a JavaScript library that provides interactive reporting features within a Web application including a range of visualisation charts. This API is used for drawing several of the interactive charts which provide additional analysis tools. Embedding such a reporting facility is a powerful technique to assist developers to perform analysis while visualising the interaction map simultaneously.

By adhering to standards and using predominantly open source technologies, the functionality of the system can be easily extended through the addition of new modules. For example, we are in the process of incorporating a clustering engine, as shown in Figure 3 to help interpret mouse trajectories by applying aggregation techniques.

\section{A Case Study}

In order to demonstrate the power of VizAnalysisTools, this section presents a case study. By examining how a user interacted with a particular map, it is 


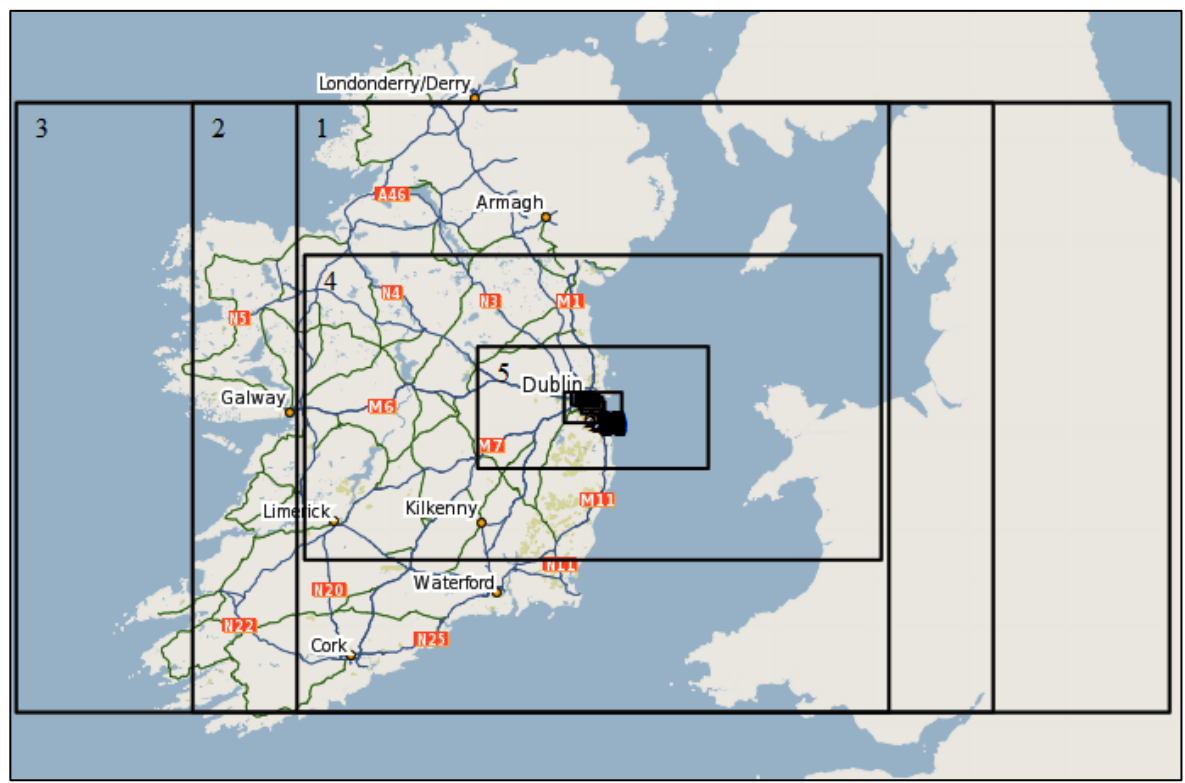

Figure 4 The succession of bounding boxes generated by a user

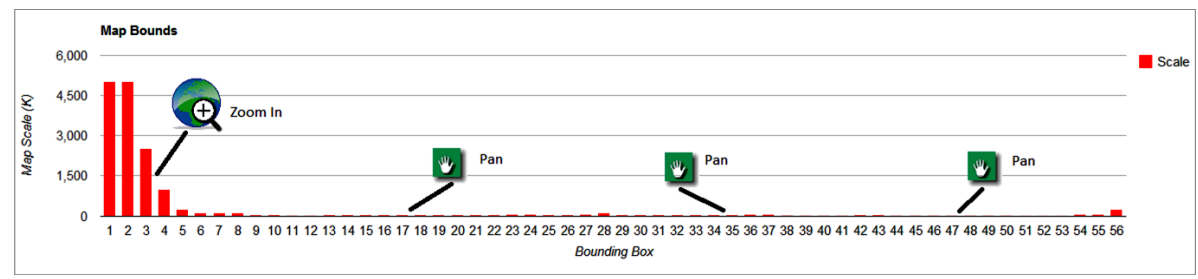

Figure 5 Variation in map scale changes based on the search task

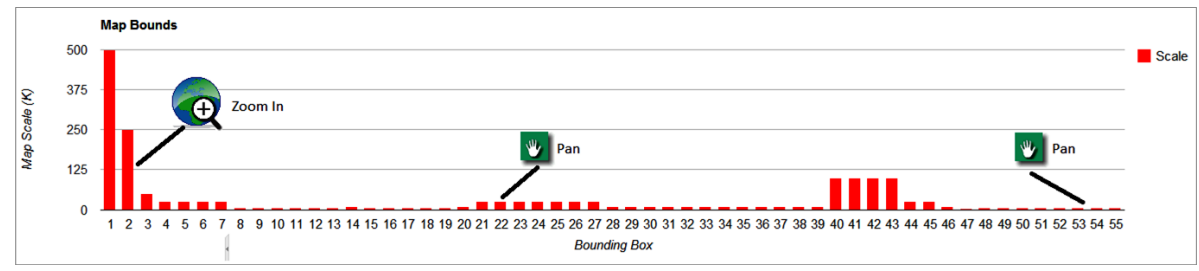

Figure 6 Variation in map scale changes based on a route finding task

possible to interpret their intentions and identify their interests with the help of Geovisualisation techniques. The user interactions on a map were recorded in the form of mouse movements, map clicks, mouse hesitations, and mouse speed at multiple map scales. 
Figure 4 presents the series of bounding boxes which the user viewed on the map. In this example it is evident that the user is interested in Ireland. The smaller boxes shown on the map indicate that the user quickly zoomed into the area of the map showing Dublin. This is a clear indication of their area of interest. Our system extracts graphs that show viewers the map scale during a user session. Zooming and pan operations are also indicated (See Figure 5 and 6 ). The graph in Figure 5 clearly shows that once the user had reached a certain zoom level, they were happy to maintain that zoom level as they panned the map. This is an indication that they were interested equally in all areas of the map at that zoom level. This is indicative of a scanning operation performed on the map as several map features are examined. It corresponds to a user seeking an overview of the entire region (Dublin). This type of behaviour is in contrast to a route finding task in which it would be expected to see a succession of zoom and pan operations as the user identifies the two areas between which they need to derive a route as highlighted in Figure 6.

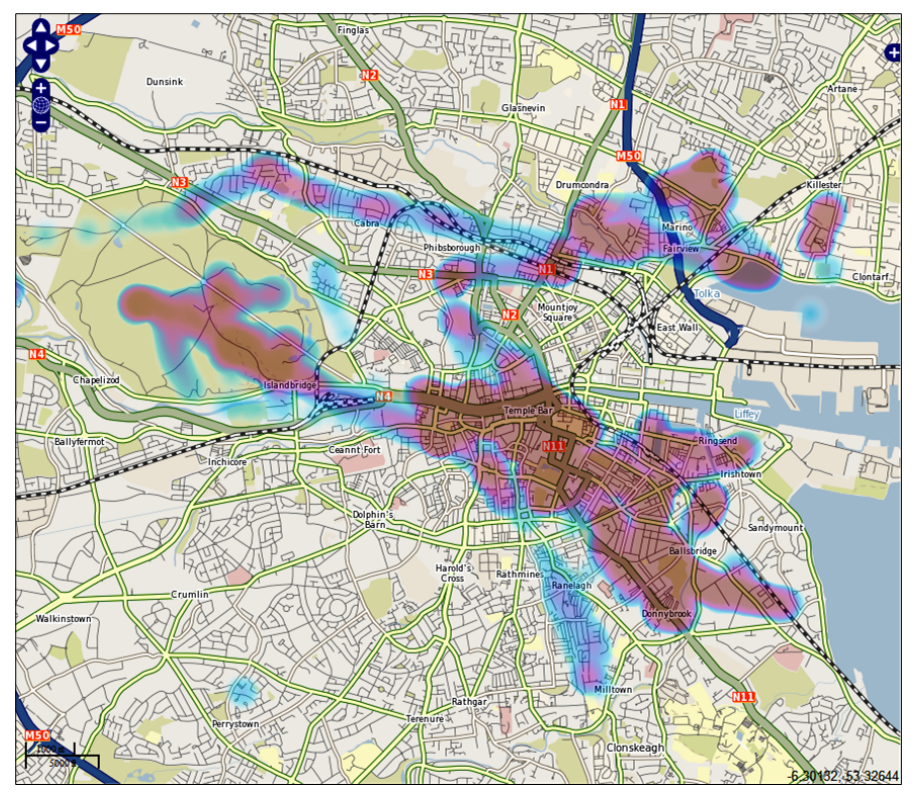

Figure 7 A heat map of user mouse activity in Dublin

Figure 7 shows a heat map of user mouse activity on the underlying map. It can be clearly seen which areas of the map had most activity. A person with background knowledge of Dublin City, would know that these regions correspond to the cultural centre of Dublin and encompass the areas of Temple Bar, Trinity College and the Georgian Quarter. Furthermore, the region of high activity seen to the west of the city is a large park, The Phoenix Park, which is where Dublin Zoo is located. Interest in these regions is indicative of a tourist visiting Dublin.

While this heat map gives an overall view of the regions visited we can also be more precise in determining user interests by examining the heat map at a lower 


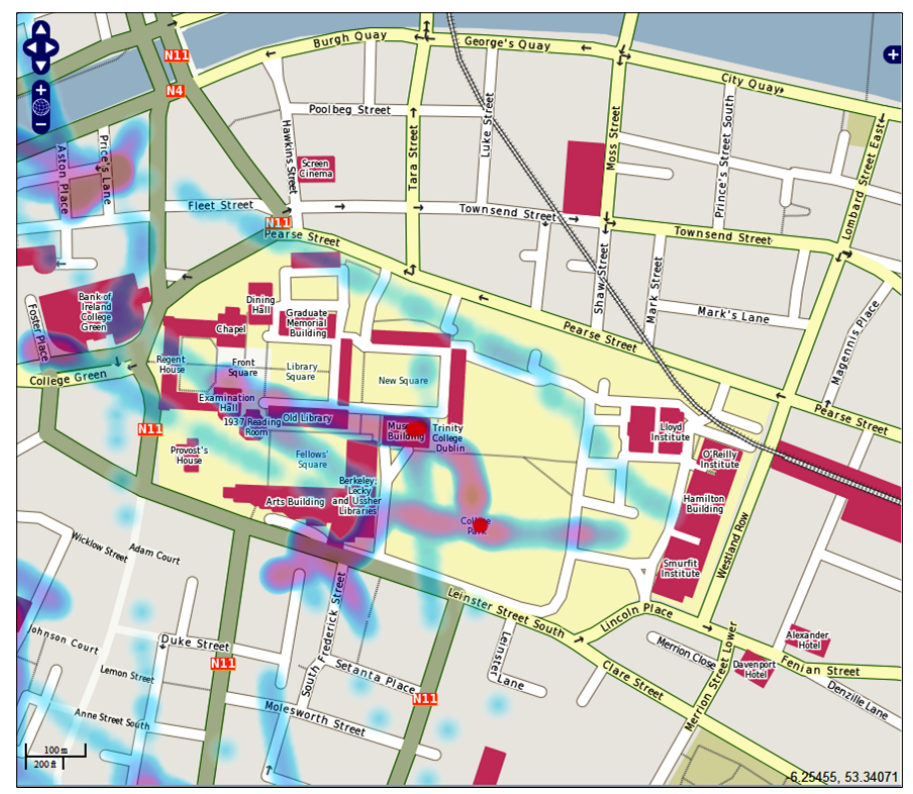

Figure 8 A heat map showing areas of interest around Trinity College Dublin

scale. Figure 8 displays the heat map for the area around Trinity College Dublin. Using this finer-grained visualisation it is possible to be more accurate regarding this particular user's areas of activity on the map. For example, there are 'hotspots' at the entrance to the college and at the museum building which, in this case, has been clicked on by the user to find more associated information. This finer grained information reveals more about the user and their interests.

In order to gain an understanding of the particular features of the map which are of interest to the user, Figure 9 demonstrates how VizAnalysisTools can be used to identify the locations on the map where the user hesitated with the mouse (shown as grey circles in which the radius corresponds to the duration of the mouse hesitation) or clicked on (shown as dots on the map).

Based on existing work, it has been shown that mouse rests are a good indicator of interest in the feature nearest the resting point (Mac Aoidh et al., 2008). The longer the hesitation period, (up to a certain threshold) the more interest the user has in that region. Similarly, a click on a map feature is an indicator of interest as they seek to obtain information corresponding to the feature. Again, given some knowledge of Dublin, it can be seen that this user clicked on several parks in the city centre. Furthermore, the mouse rested for its longest duration near some of Dublin's tourist attractions such as O'Connell Bridge and The Guinness Brewery. The graphs shown in Figure 10 depict each map and non-map event (click, pan, zoom, etc.) as a percentage of all mouse activity. This information can be used to classify the type of user. In this case pan is the predominant map activity which supports the hypothesis that this user has a general interest in the area of the map rather than being focused on a single location (in which case the click operations would be more prevalent than pan and zoom operations). 


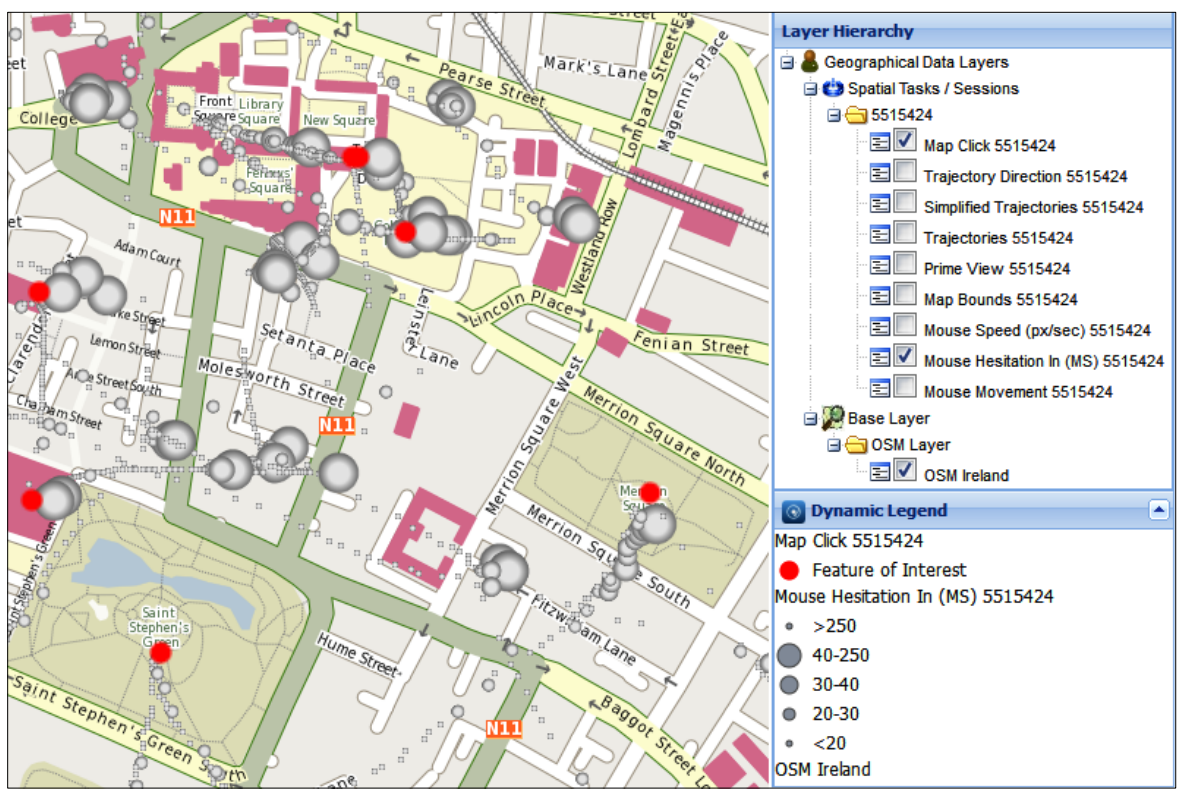

Figure 9 Mouse hesitations and mouse clicks showing user interests

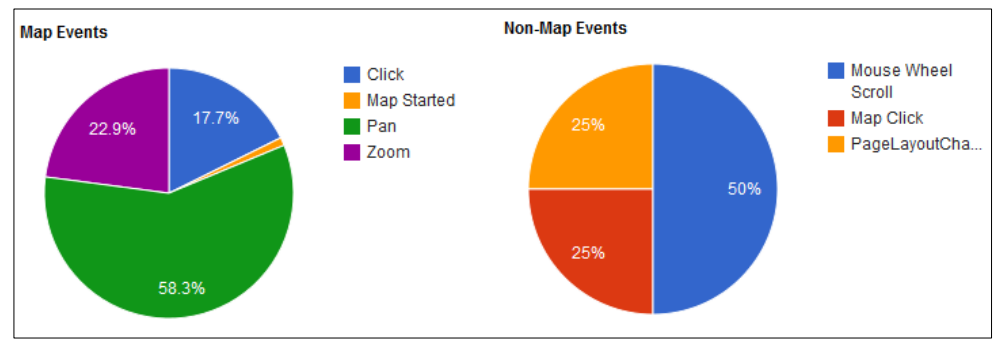

Figure 10 Pie charts showing the ratio of map and non-map events

VizAnalysisTools has given an indication that this user is potentially a tourist and indicated the areas of the city which they are attracted to and some of the map features they are interested in. Two further visualisations give more insight into the mouse activity. Figure 2 shows the speed distribution of the trajectories which the mouse followed while the user interacted with the map. In this case, the speed of the mouse is consistent throughout the entire interaction session. This can be seen both on the map where there is no colour variation of the trajectories in Figure 11, and in the accompanying plot shown to right of the map. These results correspond to a scanning action which the sequence of bounding boxes at the same zoom level also indicated.

If the user in question was familiar with the underlying geography of the map it would be expected to see faster speed over some areas of the map where the user moved quickly to a feature they were interested in (as they know its location). 


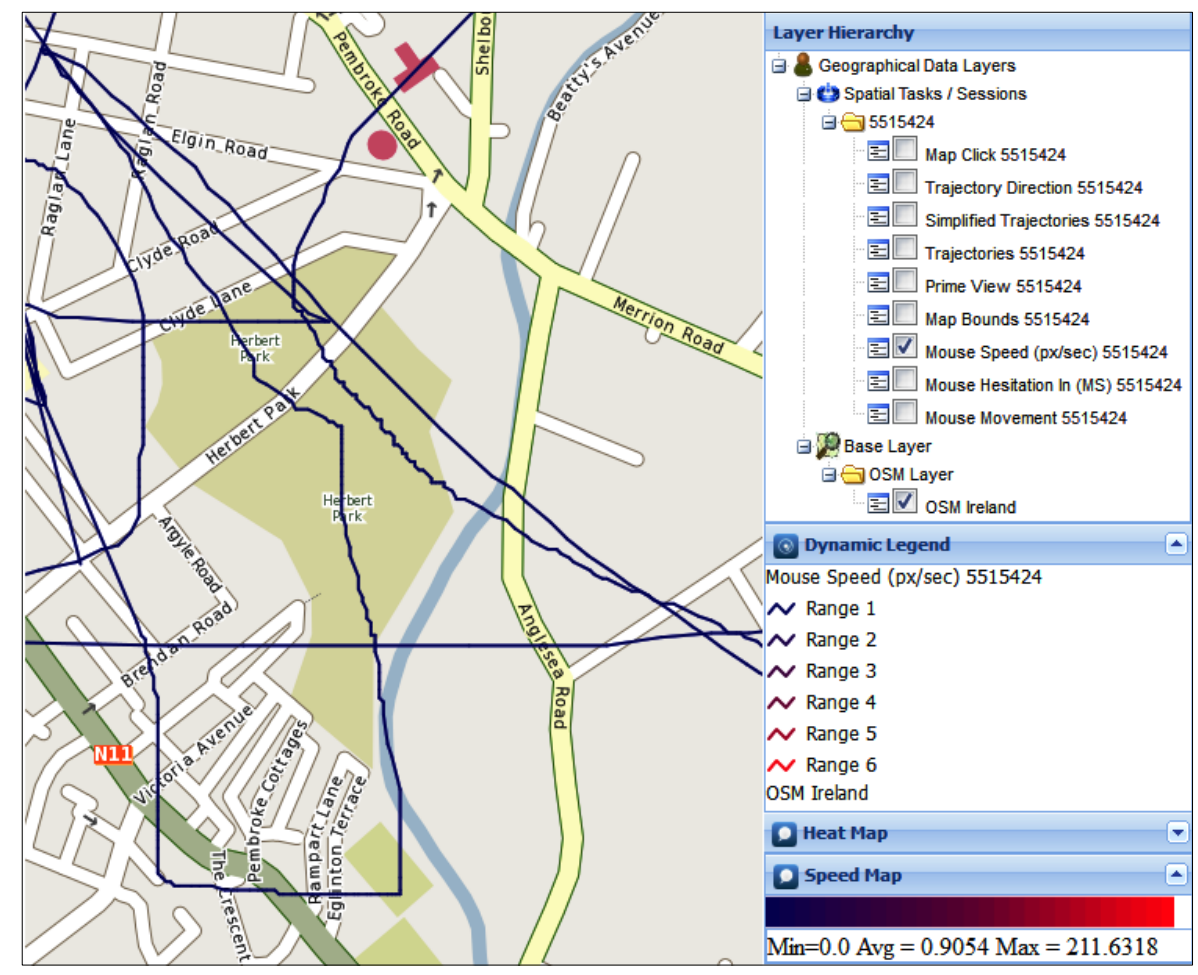

Figure 11 The speed distribution of mouse trajectories

However, in this case we can assume that this user has little knowledge of the map in question.

The case study discussed in this section illustrates how VizAnalysisTools can be used to interpret the actions of a user interacting with a Web mapping application. The knowledge gained through this process can be used to adapt user profiles and strengthen personalisation and recommendation algorithms. For example, an advertising company would be interested in finding out hot spots and areas of interests to customers.

\section{Conclusions and Future Work}

This article describes VizAnalysisTools, a tool which uses Geovisualisation techniques to interpret implicit user interactions with a Web-based GIS. Web 2.0 technology is employed to deliver an interactive interface to support Geopersonalisation by applying Geovisual Analytics to recorded mouse movement data. Such functionality can assist analysts to identify spatio-temporal patterns in mouse movement data and to explore important areas of a map. The trends, behaviours and usage patterns identified through the tool can be used within Geopersonalisation in order to reduce information overload for targeted 
recommendations. Through a case study, the visualisation techniques were shown to have a potential of gaining an insight into an individual user's behaviour.

The approach can be made more powerful by visualising interactions over multiple sessions and furthermore, visualising the interactions of several users. This type of geo-visual analysis can assist developers to improve interaction paradigms while also augmenting the information required for personalising the map data and accompanying tools.

When multiple users and multiple sessions are considered, cluttering and occlusion of the map overlays may occur. This makes interpretation of the data difficult, which visualisation alone is unable to handle. To overcome this, it is important to assist analysts to identify patterns and trends within the data being studied by extending the functionality to include aggregation and clustering techniques (Andrienko and Andrienko., 2010). We are incorporating a clustering engine, in the Web architecture which provides clustering and aggregation Web services to group similar mouse trajectories based on spatial and temporal similarity metrics (Braga et al., 2012). There are several approaches for aggregating movement data and measuring the similarity of trajectories. The approaches vary, depending on the analyst's focus. For example, trajectories can be aggregated based on their similarity in geographic, temporal or attribute space. We will investigate several approaches.

To gain further insight into user behaviour, there are other visualisation techniques that can be explored. In particular the use of a space-time cube (Hägerstrand, 1970) will enable analysts to clearly see the temporal ordering and the sequence in which mouse events occurred. Focus maps (Zipf and Richter, 2002) are another pertinent visual technique that can be applied to display the features of interest to the analyst by rendering visual styles and by making other map portions (less relevant) faded. Fading techniques are also closely related to the focus maps but have the advantage of accounting (at least in part) for the temporal dimension. The Atlas of Switzerland (Oberholzer and Hurni, 2000), is an example of this where a map or aerial photography gradually become faded at a given time moment while the other portions of the map become visible.

Fisheye view is a powerful Geovisual technique that focuses the area of interest by applying a distance function. Yang et al. (2003) applied fisheye views and the fractal views techniques to visualise large category maps for Internet browsing. Fisheye views adopt the distortion approach to enlarge areas of interest while fractal views work on an information filtering approach to control the flow of information. We find these two techniques relevant in our domain. In particular a combined approach including a fisheye view irrespective of map scale, along with fractal view and focus map techniques would provide an insight into user behaviour patterns.

Finally, the preliminary findings and evaluation conducted so far have shown good results. However, a more comprehensive evaluation including a usability study is needed. This represents an important element of our future work. 


\section{Acknowledgements}

Research presented in this paper was funded by a Strategic Research Cluster grant (07/SRC/I1168) by Science Foundation Ireland under the National Development Plan. The authors gratefully acknowledge this support.

\section{References}

Andrienko, G. and Andrienko., N. (2010), "A general framework for using aggregation in visual exploration of movement data", The Cartographic Journal , Vol. 47(1), Maney Publishing, pp. 22-40.

Andrienko, G., Andrienko, N., Jankowski, P., Keim, D., Kraak, M., MacEachren, A. and Worbel, S. (2007), "Geovisual analytics for spatial decision support: Setting the research agenda", International Journal of Geographical Information Science, Vol. 21(8), London: Taylor \& Francis, pp. 839-858.

Andrienko, G., Andrienko, N. and Wrobel, S. (2007), "Visual Analytics tools for analysis of movement data", ACM SIGKDD Explorations Newsletter, Vol. 9, ACM, pp. 3846.

Arroyo, E., Selker, T. and Wei, W. (2006), Usability tool for analysis of Web designs using mouse tracks, in 'CHI'06 Extended Abstracts on Human Factors in Computing Systems', ACM, pp. 484-489.

Auer, S., Lehmann, J. and Hellmann, S. (2009), LinkedGeoData: Adding a spatial dimension to the web of data, in 'International Semantic Web Conference', pp. 731746.

Ballatore, A., McArdle, G., Kelly, C. and Bertolotto, M. (2010), RecoMap: An interactive and adaptive map-based recommender, in 'Proceedings of the 2010 ACM Symposium on Applied Computing', ACM, pp. 887-891.

Braga, R. B., Tahir, A., Bertolotto, M. and Martin, H. (2012), Clustering user trajectories to find patterns for social interaction applications, in ' 11 th International Conference on Web and Wireless Geographical Information Systems (W2GIS'12)', W2GIS '12, Lecture Notes in Computer Science - Springer. 7236, Naples, Italy.

Chen, C. (2010), "Information visualization", Wiley Interdisciplinary Reviews: Computational Statistics, Vol. 2(4), Wiley Online Library, pp. 387-403.

Curbera, F., Duftler, M., Khalaf, R., Nagy, W., Mukhi, N. and Weerawarana, S. (2002), "Unraveling the Web services web: An introduction to SOAP, WSDL, and UDDI", IEEE Internet Computing, Vol. 6(2), pp. 86-93.

Hägerstrand, T. (1970), "What about people in regional science?", Papers in Regional Science, Vol. 24(1), Springer, pp. 6-21.

Kisilevich, S., Mansmann, F., Bak, P., Keim, D. and Tchaikin, A. (2010), Where would you go on your next vacation? A framework for visual exploration of attractive places, in 'Proceedings of Second International Conference on Advanced Geographic Information Systems, Applications, and Services (GEOPROCESSING)', IEEE, pp. 21-26.

Mac Aoidh, E., Bertolotto, M. and Wilson, D. (2008), "Understanding geospatial interests by visualizing map interaction behavior", Information Visualization, Vol. 7(3), Nature Publishing Group, pp. 275-286.

Mac Aoidh, E., McArdle, G., Petit, M., Ray, C., Bertolotto, M., Claramunt, C. and Wilson, D. (2009), "Personalization in adaptive and interactive GIS", Annals of GIS , Vol. 15(1), Taylor \& Francis, pp. 23-33. 
McArdle, G., Ballatore, A., Tahir, A. and Bertolotto, M. (2010), An open-source Web architecture for adaptive location based services, in 'Proceedings 14th International Symposium on Spatial Data Handling (SDH), The International Archives of the Photogrammetry, Remote Sensing and Spatial Information Sciences, Hong Kong', Vol. XXXVIII / 2, pp. 296-301.

Mueller, F. and Lockerd, A. (2001), Cheese: Tracking mouse movement activity on websites, a tool for user modeling, in 'CHI'01 Extended Abstracts on Human Factors in Computing Systems', ACM, pp. 279-280.

Oberholzer, C. and Hurni, L. (2000), "Visualization of change in the interactive multimedia atlas of Switzerland", Computers \& Geosciences , Vol. 26(1), Elsevier, pp. $37-43$.

Oreilly, T. (2007), "What is Web 2.0", Design Patterns and Business Models for the Next Generation of Software, Vol. 1, p. 17. Available at SSRN: http://ssrn.com/abstract=1008839. Last accessed March, 2011.

Tahir, A., McArdle, G., Ballatore, A. and Bertolotto, M. (2010), Collaborative filteringA group profiling algorithm for personalisation in a spatial recommender system, in 'Proceedings Geoinformatik, Kiel, Germany', pp. 44-50.

Tahir, A., McArdle, G. and Bertolotto, M. (2011), A web-based visualisation tool for analysing mouse movements to support map personalisation, in 'Proceedings of the First International Workshop on Spatial Information Modeling, Management and Mining (SIM3) (in conjunction with DASFAA2011)', LNCS, Springer, pp. 132-143.

Thomas, J. and Cook, K. (2005), Illuminating the path: The research and development agenda for visual analytics, IEEE Computer Society.

Yang, C., Chen, H. and Hong, K. (2003), "Visualization of large category map for Internet browsing", Decision Support Systems, Vol. 35(1), Elsevier, pp. 89-102.

Zipf, A. and Richter, K. (2002), "Using focus maps to ease map reading", Künstliche Intelligenz, Vol. 4(2), Citeseer, pp. 35-37. 